\title{
We Are about to Soar
}

Jong-Ho Park

Editor-in-Chief, Journal of Neurosonology and Neuroimaging; Department of Neurology, Myongji Hospital, Goyang, Korea

First of all, it is a great honor to assume a position of editor-in-chief in the official Journal of Korean Society of Neurosonology (KSN). It has been about a decade since the foundation of KSN in 2009. On behalf of the KSN, I would like to express my sincere gratitude to the senior executive committee members for a prolonged period of passionate and devotional responsibility which has led to a remarkable development of the KSN.

Looking backward, however, we should acknowledge that the Journal of Neurosonology, a key representative of the KSN has been sedentary state quantitatively as well as qualitatively. The status and prestige of KSN also depends on the high quality activity of its official journal.

Here, I gladly inform that the journal is renamed to the Journal of Neurosonology and Neuroimaging (JNN). In the strict sense, this is not a new journal; this means a revolution of the previously recognized subdivision journal into a high standard special journal with the better quality. The JNN is a peer-reviewed, open-access official journal whose main aim is to offer the recent advances about all aspects of ultrasound and neuroimaging in the field of stroke, cerebrovascular or other neurological diseases for clinicians and researchers that will improve knowledge of clinical practice, education, and academic research. The JNN offers full coverage of all the relevant stroke or cerebrovascular aspects of neurosonology including transcranial Doppler, carotid duplex ultrasound, peripheral ultrasound, and of neuroimaging such as MRI, CT, SPECT, or PET. Specifically, the JNN includes neuroimaging marker or sign (i.e., atherosclerosis, small-vessel disease, acute ischemia, etc.) related studies associated with predicting outcome. It is also interested in the field of peripheral neuropathy based on ultrasound or imaging. Basic sciences can be welcome if the results are closely relevant to clinical practice.

As you have already recognized the new journal cover in print, we have enforced the full model change of the journal. The figure in the hard cover means a symbol of brain reflecting color ultrasound wave. We are also working to create structural e-submission site (https://www.j-nn.org/) independently as well as in the KSN homepage. To increase the numbers of submission, publication, to enrich quality thereby leading to expand the origin of citations, I think publishing in English and registration of our journal in PubMed Central in future are at least the fundamental requirements, to which I am seriously looking forward in future.

The JNN is currently published two times a year on the last day of June and December, but we hope to increase the number of publications after a successful revolution. A substantial take-off of the JNN depends on your active inter-
Received: June 5, 2018 Accepted: June 12, 2018

Address for correspondence: Jong-Ho Park

Department of Neurology, Myongji Hospital, 55 Hwasuro 14beon-gil, Deogyang-gu, Goyang 10475, Korea Tel: +82-31-810-5460 Fax: +82-31-969-0500 E-mail: neurotector.jhp@ gmail.com 
ests, therefore I hope you don't hesitate to submit your valuable works to the JNN. We also welcome valuable papers from other department including neuroradiology or neurovascular surgery.

Finally, I would like to appreciate the editorial board members, and the executive committee members from the president of KSN Yong-Jae Kim for their enormous support and expect that their veritable contributions will help shed the JNN into light. 